\title{
ANÁLISIS DE DATOS COMPOSICIONA- LES PARA EL MONITOREO DEL DES- EMPEÑO DE ESTUDIANTES DE UNA CARRERA UNIVERSITARIA DE GRADO. EL CASO DE INGENIERÍA EN AGRIMENSURA DE LA UNIVERSIDAD NACIONAL DEL NORDESTE
}

Romero, J. L. ${ }^{1}$; Caputo, L. ${ }^{2}$; Porcel, E. ${ }^{3}$; Ferrari, Elvira E ${ }^{4}$.

\section{RESUMEN}

En este trabajo se describe el desempeño de los estudiantes que cursaron las asignaturas del Plan de Estudios de la carrera de Ingeniería en Agrimensura, de la Facultad de Ciencias Exactas y Naturales y Agrimensura de la Universidad $\mathrm{Na}-$ cional del Nordeste (FACENA-UNNE), en el periodo 2011-2014. La información del resultado del cursado de dichas asignaturas, se analizó mediante la técnica de Datos Composicionales. Se observó que las mayores deficiencias en el rendimiento académico se presentan en las asignaturas de Ciencias Básicas, en las cuales los porcentajes de regularización no superan el 50\%. Esta situación se registra fuertemente en las asignaturas de primer año del primer cuatrimestre, siendo elevado el porcentaje de alumnos libres por asistencia. Esta in- formación permitirá apoyar el proceso de monitoreo de la implementación del plan de estudios, actividad comprometida en el proceso de acreditación de la carrera.

Palabras Clave: Rendimiento académico, Nivel Universitario, Datos composicionales, Gráficos ternarios, Envolvente convexa.

\section{Introducción}

El objetivo del presente trabajo es describir el desempeño de los estudiantes que cursaron las asignaturas correspondientes a los cuatro primeros años de la carrera de Ingeniería en Agrimensura de la Facultad de Ciencias Exactas y Naturales y Agrimensura (FACENA), durante los años 2011 a 2014, a fin de aportar información para el monitoreo de la implementación del plan de estudios, actividad compro-

Facultad de Ciencias Exactas y Naturales y Agrimensura. Universidad Nacional del Nordeste

Avda. Libertad 5450. Corrientes. 3400

${ }^{1}$ Profesor de Matemática; joseluisromero@live.com.ar

${ }^{2}$ Especialista en Docencia universitaria; proflcaputo@gmail.com

${ }^{3}$ Magister en Estadística Aplicada; porcelfel@arnet.com.ar

${ }^{4}$ Agrimensora; elviraeferrari@yahoo.com.ar 
metida en el proceso de acreditación de la carrera. Se hace entonces necesario evaluar los indicadores de rendimiento académico, en particular aquellos que resultan más preocupantes para la carrera tales como el desgranamiento de los alumnos de primer año y su bajo rendimiento, situaciones que afectan la permanencia y terminación exitosa de sus estudios. Esta problemática afecta a todas las carreras que integran la oferta curricular de la Facultad y se agudiza en el caso de las ingenierías: Puede señalarse que, en un trabajo previo se estableció que, de los 519 ingresantes a Ingeniería Electrónica de las cohortes 2001 a 2005, sólo 38 alumnos habían cursado y aprobado todas las asignaturas del Ciclo Básico en el período 2001 - 2010 [3]. Asimismo, se ha determinado que en las carreras Ingeniería en Electrónica e Ingeniería Eléctrica, los largos períodos de tiempo para concluir dicho Ciclo Básico son consecuencia de la dificultad de aprobar las asignaturas de las ciencias básicas (Matemática y Física) [2].

Además de las dos carreras de Ingeniería mencionadas, en la FACENA se puede cursar la carrera de Ingeniería en Agrimensura. Su plan de estudios se puso en vigencia a partir de 2011, y está organizado por asignaturas, con una duración teórica de 5 años lectivos. Consta de 33 asignaturas cuatrimestrales y 1 asignatura anual, un examen de idiomas (denominado Inglés Técnico), más un Trabajo Final y una Práctica Profesional Supervisada previstos en el último año de estudios. Las asignaturas están distribuidas en cuatro Bloques Temáticos: Ciencias Básicas, Tecnologías Básicas, Tecnologías Aplicadas y Complementarias. Las Asignaturas de Ciencias Básicas están ubicadas mayoritariamente en el primer año y primer cuatrimestre de segundo año de la carrera, las de Tecno- logías Básicas, preferentemente, en el segundo cuatrimestre de segundo año y los restantes bloques, a partir de tercer año.

En este trabajo, no se incluye el análisis del desempeño en el bloque de asignaturas Complementarias, dado que se cursan en su mayoría en el último año de estudios, el cual se está cursando por primera vez en el presente año lectivo.

\section{Metodología}

Como información de base, se utilizó el resultado del dictado de las asignaturas de la carrera de Ingeniería en Agrimensura, disponible en el sistema de gestión de alumnos de la FACENA: SIU Guarani, información suministrada por el Departamento Estudios de la Facultad, que registra si cada alumno se inscribió pero no cursó la asignatura, si la regularizó o promocionó, si quedó libre por parciales o por asistencia. Para cada asignatura se obtuvo el número de alumnos en esas categorías.

El listado de las asignaturas con su ubicación en cada año y cuatrimestre y el bloque temático al que pertenece cada una de ellas, se presenta en la Tabla 1 .

En este trabajo, se analizaron:

1) E1 desempeño de los alumnos en las asignaturas de primer año desde 2011 a 2014, y su evolución a través del tiempo. 2) El desempeño en las asignaturas de segundo y tercer año, y 3) El desempeño en las asignaturas según bloque temático.de pertenencia.

Las unidades de análisis son cada una de las asignaturas que se cursaron en dichos años y se utiliza como indicador del rendimiento académico de los estu- 
Tabla 1. Asignaturas de la carrera. Ubicación, bloque temático y código

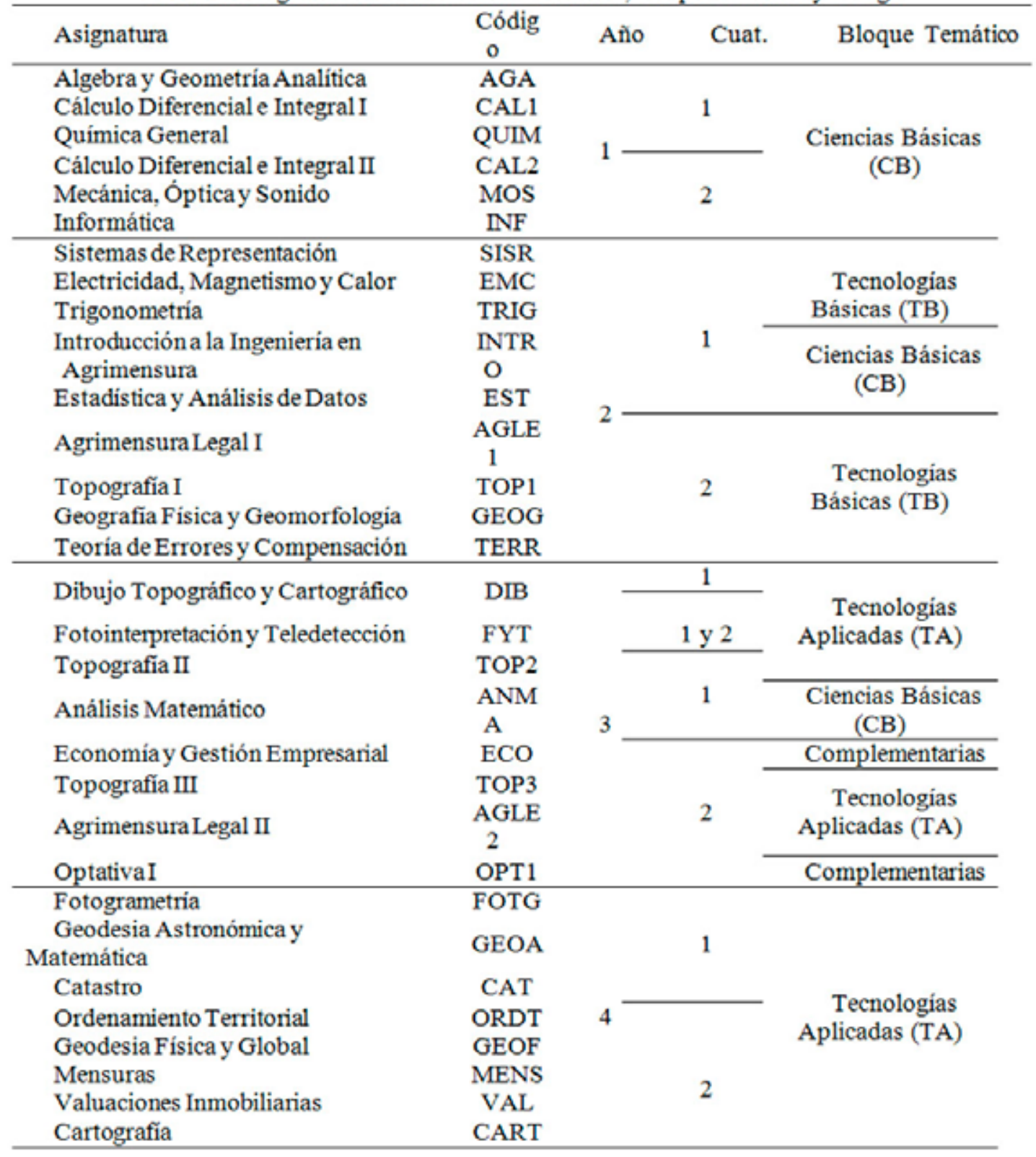

diantes en las asignaturas que cursaron, su desempeño durante dicho cursado.

La información antes citada fue estudiada mediante el análisis de datos composicionales [3]. Los datos composicionales son vectores $\mathrm{X}$ de componentes no negativas $\mathrm{x}_{\mathrm{i}}$, con $\mathrm{i}=1, \ldots, \mathrm{D}$, que representan proporciones de un total, y cumplen la condición que
$\mathrm{x}_{1}+\mathrm{x}_{2}+\ldots+\mathrm{xD}=1$ (o 100 , si los datos se expresan en porcentaje). Este tipo de análisis es útil cuando interesa estudiar datos expresados en forma de proporción o porcentaje.

En este trabajo, para cada asignatura, el vector $\mathrm{X}$ de resultados, tiene las componentes: 
$\mathrm{x}_{1}: \%$ de alumnos libres por asistencia; $\mathrm{x}_{2}: \%$ de alumnos libres por parciales; $\mathrm{x}_{3}: \%$ de alumnos que promocionaron $\mathrm{y} \mathrm{x}_{4}: \%$ de alumnos que regularizaron.

El desempeño de los alumnos en las asignaturas, se analizó mediante el vector $\mathrm{X}^{\prime}=\left(\mathrm{x}_{1}, \mathrm{x}_{2}, \mathrm{x}_{3}+\mathrm{x}_{4}\right)$ siendo el $100 \%$ el total de alumnos que cursaron cada asignatura. Los resultados se presentan mediante gráficos ternarios y cápsulas convexas.

Los gráficos ternarios se utilizan para analizar la importancia porcentual de las categorías en que puede presentarse una variable. En este caso se utilizaron gráficos en los que las escalas de las componentes: libres por asistencia $\left(\mathrm{x}_{1}\right)$, libres por parciales $\left(\mathrm{x}_{2}\right)$ y promovidos + regulares $\left(\mathrm{x}_{3}+\mathrm{x}_{4}\right)$ están orientadas de menor a mayor (0\% a $100 \%)$ en el sentido de las agujas del reloj. La pertinencia de este gráfico para representar vectores tridimensionales en el plano, se basa en la propiedad de los triángulos equiláteros que dice: Las paralelas a cada lado, trazadas por un punto $\mathrm{P}$ del triángulo, determinan sobre cada uno de ellos, segmentos cuya suma de us longitudes es constante e igual a la longitud del lado [5]. Así pues, el conjunto de puntos representados en un gráfico ternario, determinan un espacio ( $\mathrm{Fi}^{-}$ gura1a), denominado espacio simplex [1].
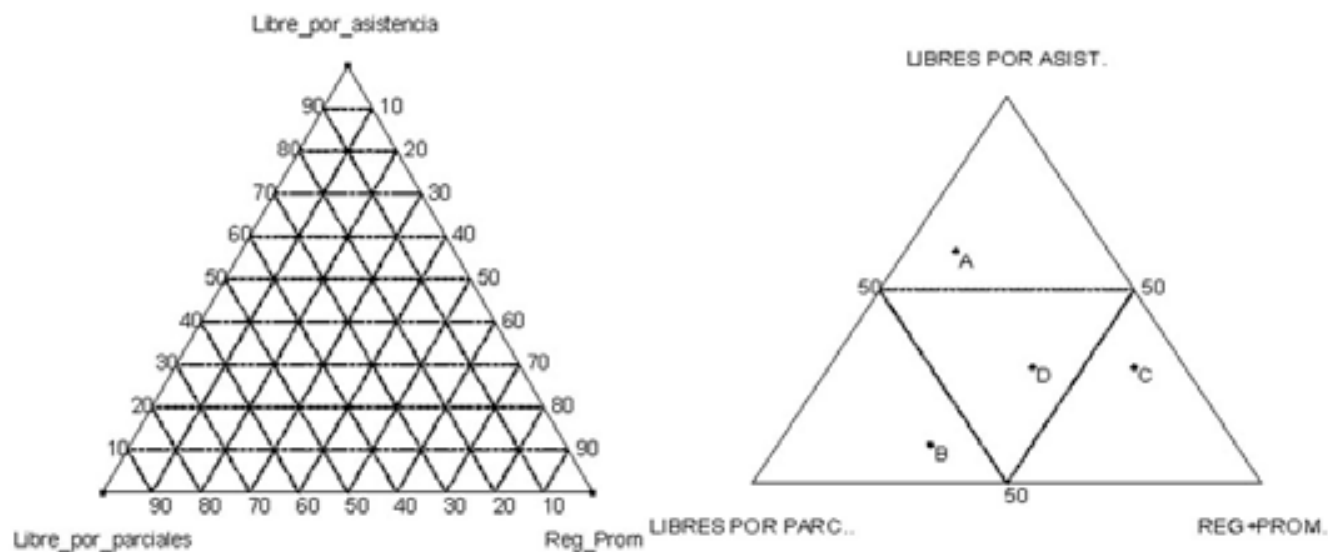

Fig. 1. Gráfico ternarios con divisiones en el $10 \%$ (a) y $50 \%$ (b) de la escala de cada eje.

Para una mejor visualización, en adelante los gráficos se presentan sin las divisiones intermedias de la escala de cada eje, tal como se ve en la Figura 1b

Entre las asignaturas objeto de estudio, es posible distinguir cuatro grupos:

- El conformado por aquellas asignaturas en que predominan los alumnos libres por parciales (no regularizan por no aprobar las evaluaciones implementadas durante el cursado), representadas por los puntos ubicados en el triángulo inferior izquierdo (asignatura B en la Figura 1b).

- El conformado por aquellas asignaturas en que predominan los alumnos libres por asistencia (no alcanzan el porcentaje mínimo de asistencia a clases), representadas por los puntos ubicados en el triángulo superior (asignatura A en la Figura 1b).

- E1 conformado por aquellas asignaturas en que predominan los alumnos regulares o aprobados por promoción, representadas por los puntos 
ubicados en el triángulo inferior derecho (asignatura $\mathrm{C}$ en la Figura 1b).

- El conformado por aquellas asignaturas en que los porcentajes de alumnos son similares en las tres categorías mencionadas; dichas asignaturas están representadas por los puntos ubicados en el triángulo central (asignatura D en la Figura 1b).

La cápsula o envolvente convexa de un subconjunto $\mathrm{X}$ de un espacio $\mathrm{n}$ - dimensional $\mathrm{V}$, se define como la intersección de todos los subconjuntos convexos de $\mathrm{V}$ que contienen a X. En este trabajo para analizar el desempeño de los alumnos en el cursado de las asignaturas según el año de la carrera a la que pertenecen, se hallaron las cápsulas convexas de los conjuntos de asignaturas de cada año de estudios de la misma [5].

\section{Resultados}

En 2011 en las asignaturas de formación básica del primer cuatrimestre de primer año, se observó una tendencia a que los alumnos quedaran libres por asistencia.
(51\%) en Cálculo Diferencial e Integral I y Química General con un bajo porcentaje de regularización en ambas (24\%); en cambio, en Álgebra y Geometría Analítica quedaron libres un $60 \%$ de los alumnos. En tanto en el segundo cuatrimestre Cálculo Diferencial e Integral II registró un 50\% de alumnos que quedaron libres y $50 \%$ de alumnos que regularizaron, y en las restantes asignaturas de ese cuatrimestre el porcentaje de alumnos que regularizaron fue superior al $77 \%$.

En 2012, en las asignaturas del primer cuatrimestre mejoraron los porcentajes de regularización de Química General (50\%) y de Cálculo Diferencial e Integral I (43\%), en tanto que en Álgebra y Geometría Analítica se mantuvo igual al registrado en 2011. En las del segundo cuatrimestre, Mecánica, Óptica y Sonido y Cálculo Diferencial e Integral II disminuyeron su rendimiento ya que regularizaron el $40 \%$ y el $36 \%$ de los alumnos, respectivamente. En Sistemas de Representación e Informática se mantuvo el buen desempeño registrado en el año anterior (Figura 2).
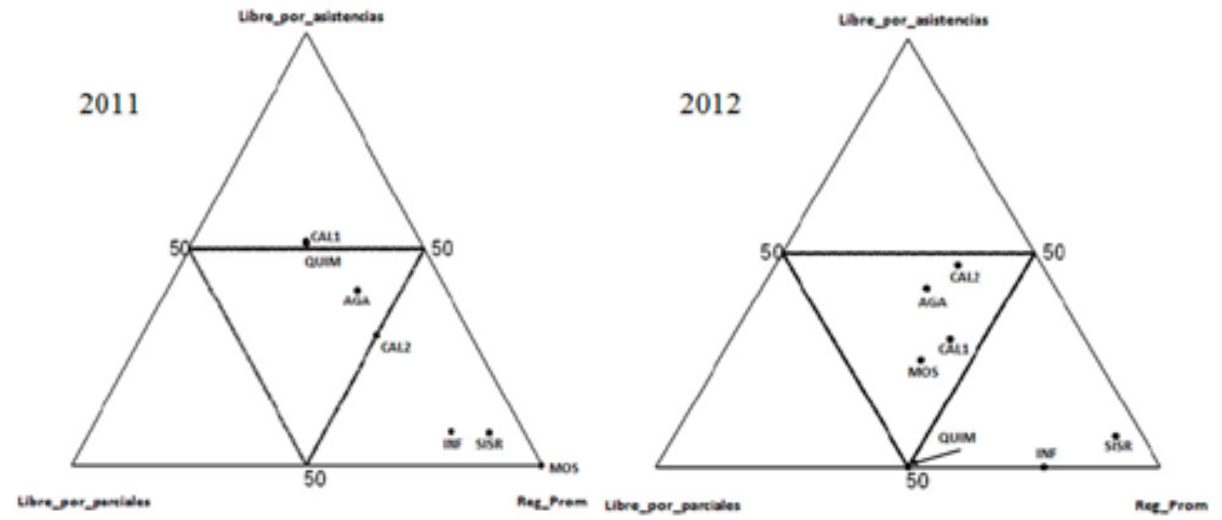

Fig. 2. Rendimiento académico de asignaturas de primer año en 2011 y 2012.

En 2013, las asignaturas del primer cuatrimestre se mantuvieron dentro del triángulo central, a excepción de Química Ge- neral, cuyo buen rendimiento disminuyó al $34 \%$ y presentó un alto porcentaje de libres por asistencia (56\%). En el segundo cua- 
trimestre, Cálculo Diferencial e Integral II mejoró su porcentaje de alumnos regulares $(82 \%)$, permaneciendo las restantes asignaturas en el triángulo de buen rendimiento.

En 2014, en el primer cuatrimestre, mientras Química General evidencia elevados porcentajes de libres por asistencia (49\%), Álgebra y Geometría Analítica y Cálculo Diferencial e Integral I se despla- zaron hacia el triángulo en que predominan los porcentajes de alumnos libres por parciales. (48\% y 45\%, respectivamente). En cambio en el segundo cuatrimestre, en Mecánica, Óptica y Sonido aumentó, respecto a 2013, el porcentaje de alumnos libres por parciales (52\%), permaneciendo las restantes asignaturas en el triángulo de buen rendimiento académico. (Figura 3).
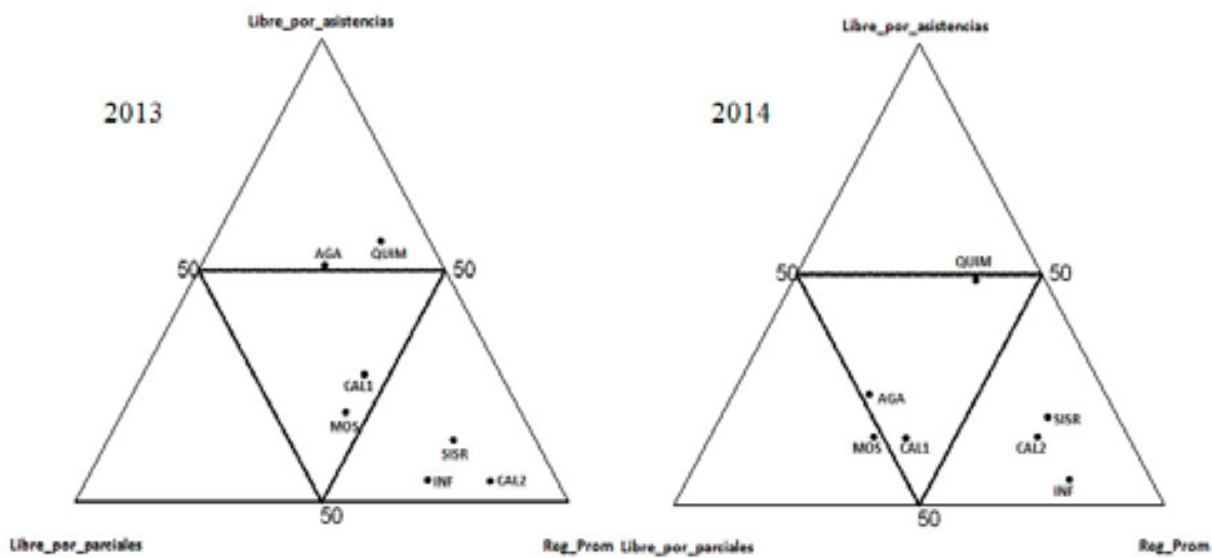

Figura 3. Rendimiento académico de asignaturas de primer año en 2013 y 2014.

Analizando ahora el desempeño por asignatura mediante las cápsulas convexas se observa que las asignaturas del primer cuatrimestre se mantuvieron en general en el triángulo de rendimientos intermedios, pero con tendencia a que los alumnos queden libres por asistencia. En las asignaturas del segundo cuatrimestre, Sistemas de Representación e Informática se mantuvieron en todos estos años en el triángulo en que predominan los porcentajes de alumnos que regularizaron $\mathrm{o}$ promocionaron las asignaturas, mientras que Mecánica, Óptica y Sonido se mantuvo en el triángulo central y Cálculo Diferencial e Integral II osciló entre este y

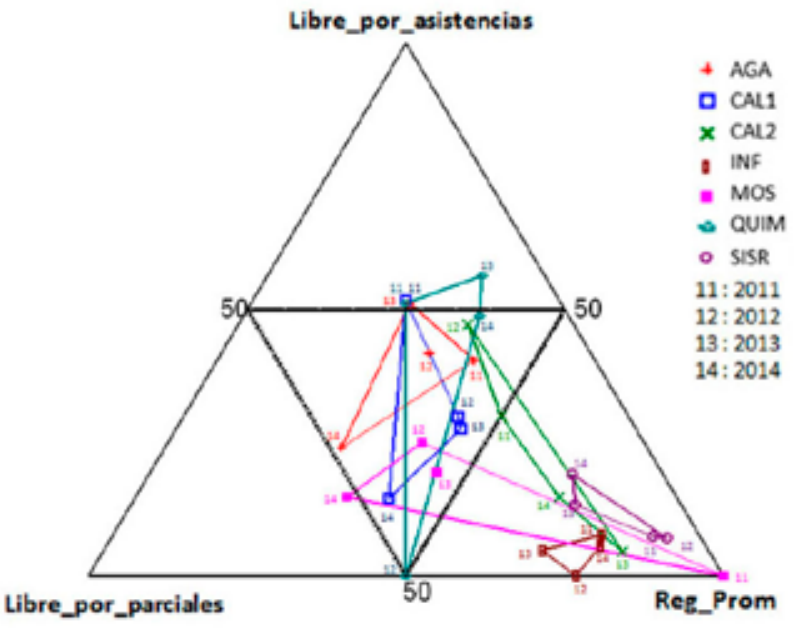

Figura 4. Cápsulas convexas de asignaturas de primer año en los años 2011 a 2014 el de buen rendimiento (Figura 4). 
En relación a las asignaturas de segundo año, que cursaron los alumnos en los años 2012 a 2014, se observa que, en todos los años, todas ellas se mantuvieron en el triángulo de buen rendimiento, a excepción de Estadística y Análisis de datos, que en el año 2013 registró un 50\% de alumnos regulares, $29 \%$ de alumnos libres por asistencia y $21 \%$ libres por parciales (Figura 5).
En las asignaturas de tercer año, cursadas por los alumnos en 2013 y 2014 se observa que, en dicho período, todas ellas se mantuvieron en el triángulo de buen rendimiento, a excepción de Análisis Matemático que en el 2014 registra un 60\% de alumnos libres por parciales (Figura 6).

Todas las asignaturas de cuarto año, pre-

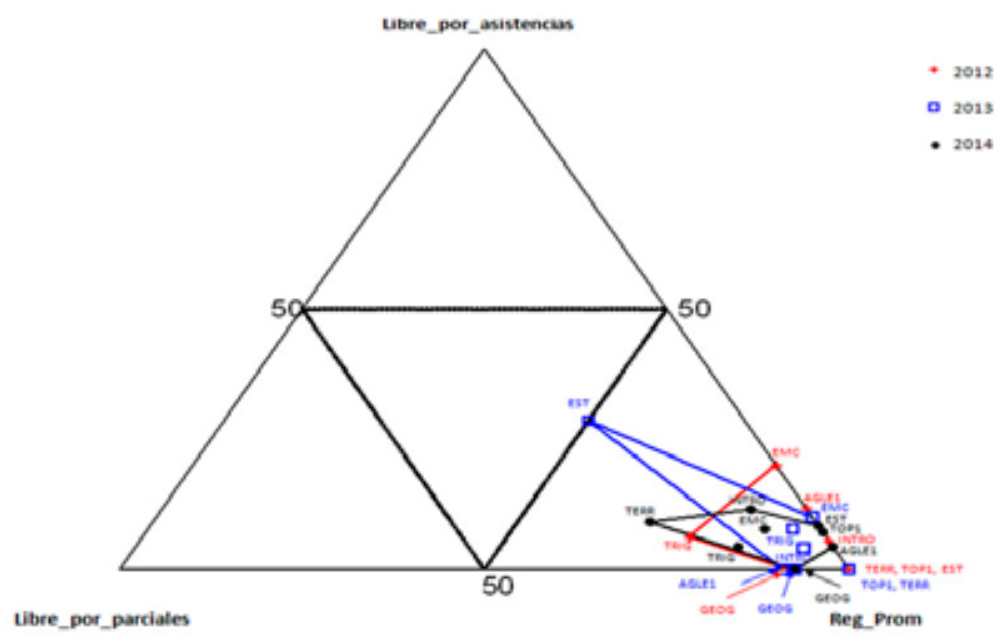

Figura 5. Cápsulas convexas de asignaturas de segundo año en los años 2012 a 2014.

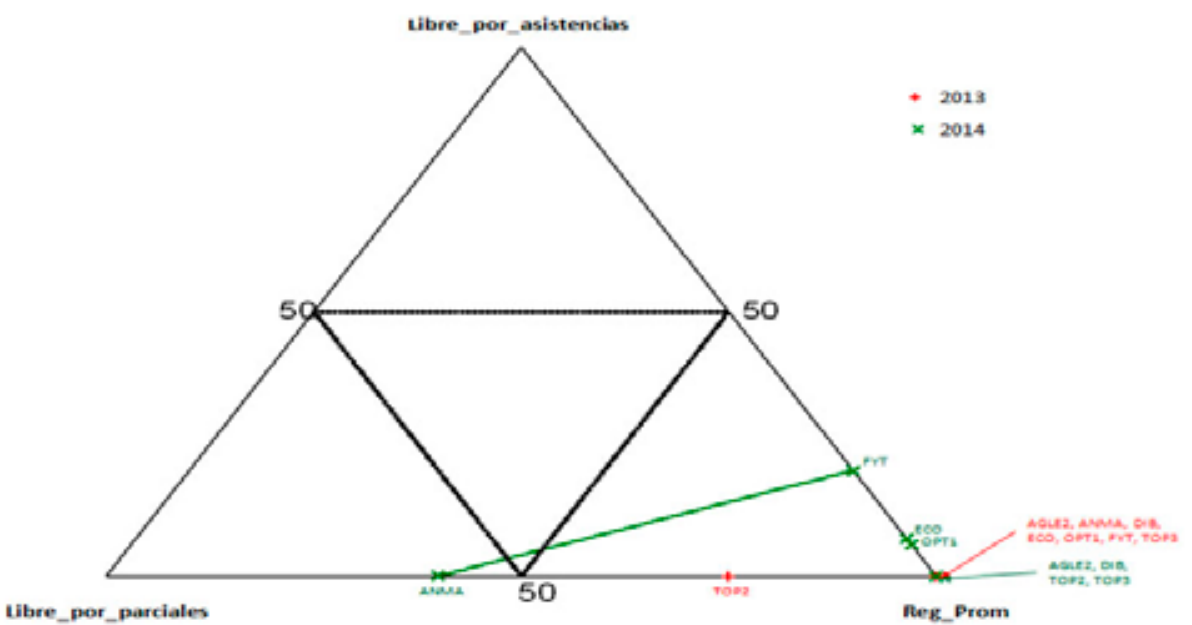

Figura 6. Cápsulas convexas. Asignaturas de tercer año. Años 2013 y 2014. 
sentaron porcentajes de regulares superiores al 90\%, dado que el número de dichos alumnos era sumamente pequeño (menos de 10).

Analizando el rendimiento de las asignaturas en función del Bloque Temático al que pertenecen, puede observarse que las asignaturas del Bloque de Ciencias Básicas registran, notablemente, peores desempeños que las de los Bloques de Tecnologías Básicas y de Tecnologías Aplicadas (Figura 7).

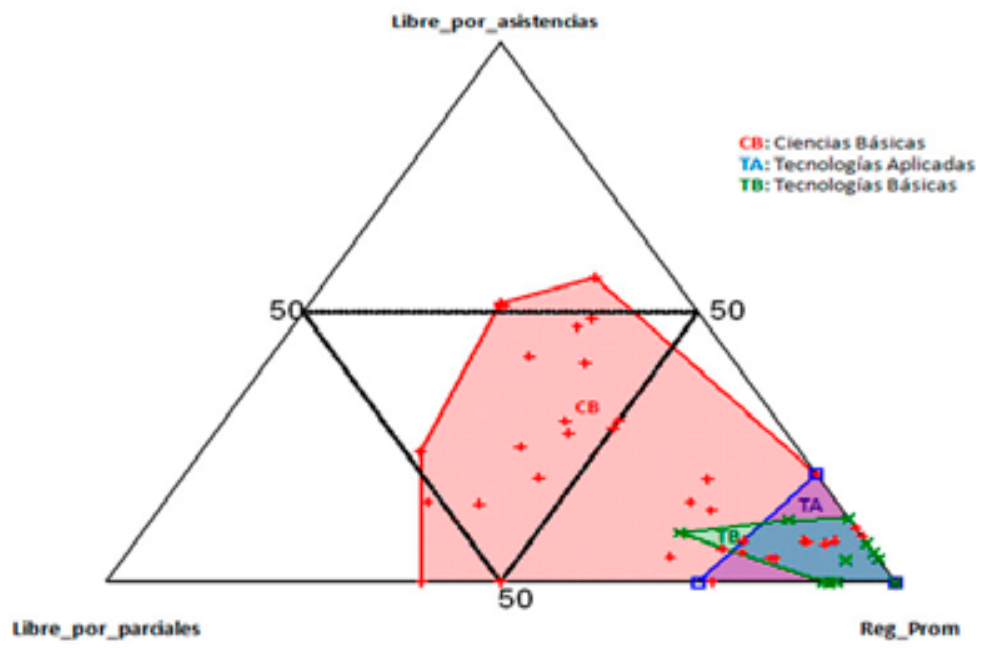

Figura 7. Desempeño de las asignaturas, clasificadas según bloques temáticos.

\section{Conclusiones y trabajos futuros}

Puede concluirse que las mayores deficiencias en el rendimiento académico se presentan en las asignaturas del bloque de Ciencias Básicas, para todos los años analizados, en el que casi todas las asignaturas se concentran en el triángulo de rendimientos intermedios, y donde los porcentajes de regularización no superan el 50\%. Esta situación se registra fuertemente en las asignaturas de primer año del primer cuatrimestre, siendo elevado el porcentaje de alumnos libres por asistencia, lo que estaría indicando que existen altos niveles de desgranamiento en dicha etapa de la carrera. Se observan también bajos rendimientos en las asignaturas de forma- ción matemática de segundo y tercer año.

Las afirmaciones hechas, no implican negar o desconocer la incidencia que en la regularización de las asignaturas tienen algunos factores institucionales: los datos que se están analizando hacen referencia a hechos ocurridos en distintos momentos históricos y en contextos institucionales diferentes (profesores, metodologías de evaluación, etc.) que no permanecen inmutables en el tiempo, por lo cual no sería adecuado utilizar estos resultados con fines predictivos, pero sí es importante su difusión en la comunidad educativa para ayudar a mejorar los mecanismos de retención de alumnos para disminuir el desgranamiento, especialmente, durante el primer año de estudios. 
La técnica de datos composicionales ha resultado adecuada para indagar respecto al desempeño de los alumnos en las asignaturas. Cabe señalar que esta misma técnica será utilizada para analizar el rendimiento aca- démico desde otras perspectivas, utilizando otros vectores composicionales tales como: (alumnos que no cursaron, libres, regulares + promovidos) o (alumnos que no cursaron, libres por parciales, libres por asistencia).

\section{REFERENCIAS}

[1] Aitchison, J. The Statistical Analysis of Compositional Data. Editorial Chapman \& Hall LTD.Londres,GranBretaña.416pp.(1986).

[2] Avalos, J.; Caputo, L.; Porcel, E.: Tiempos de aprobación de asignaturas del Ciclo Básico de Ingeniería en Electrónica de FACE$N A$. Anales del XXV Encuentro Nacional de Docentes de Investigación Operativa y XXIII Escuela de Perfeccionamiento en Investigación Operativa. Facultad de Ingeniería - UBA. CABA. Argentina. (2012).

[3] Caputo, L.; Porcel, E.; Avalos, J.: Trayectorias de formación de alumnos de las carre- ras de Ingeniería de FACENA - UNNE. Plan 2001. Anales del EMCI 2014. Facultad de Ingeniería - U.N. de Mar del Plata. Mar del Plata. Argentina.

[4] Preparata, F.P.; Hong, S.J. (1977). Convex Hulls of Finite Sets of Points in Two and Three Dimensions. Asociation for Computing Machinery. 87 - 93 pp. http://www. cs.jhu.edu/ misha/S pring14/Preparata77.pdf . Accedido: 8 de abril de 2015.

[5] Simeray, J.P. Los Gráficos al Servicio de la Empresa. (Eds): Deusto S.A. Bilbao, España. 461 pp. (1979). 